\title{
Calibration and in-orbit performance of the Argus 1000 spectrometer - the Canadian pollution monitor
}

\author{
Rajinder K. Jagpal, ${ }^{a}$ Brendan M. Quine, ${ }^{\text {abb }}$ Hugh Chesser, \\ Sanjar M. Abrarov, ${ }^{b}$ Regina Lee ${ }^{\mathrm{b}}$ \\ ${ }^{a}$ York University, Department of Physics and Astronomy, 4700 Keele Street, Toronto, \\ Ontario M3J 1L3, Canada \\ jagpal@yorku.ca, bquine@yorku.ca \\ ${ }^{\mathrm{b}}$ York University, Department of Earth and Space Science and Engineering, 4700 Keele \\ Street, Toronto, Ontario M3J 1L3, Canada \\ chesser@yorku.ca, abrarov@yorku.ca, reginal@yorku.ca
}

\begin{abstract}
Argus 1000 is a new generation miniature pollution-monitoring instrument to monitor greenhouse-gas emission from the space. Argus was launched on the CanX-2 microsatellite April 28, 2008. Operating in the near infrared and in a nadir-viewing mode, Argus provides a capability for the monitoring of Earth-based sources and sinks of anthropogenic pollution. It has 136 near infrared channels in the spectral range of 0.9-1.7 $\mu \mathrm{m}$ with an instantaneous spatial resolution of $1.25 \mathrm{~km}$. With a mass of just $228 \mathrm{~g}$ in flight-model configuration, the instrument is a demonstrator for a future micro-satellite network that can supply near-real time monitoring of pollution events in order to facilitate the detection of the sources causing climate change. In this Letter, we describe the instrument, the analysis concept behind Argus 1000 and its in-orbit performance. Recent spectral data taken over Ontario, Canada, are presented.
\end{abstract}

Keywords: infrared spectroscopy, infrared detector, infrared measurement, remote sensing, air pollution, calibration.

\section{INTRODUCTION}

The Argus 1000 is a micro-spectrometer with a mass of $228 \mathrm{~g}$ and dimensions of $45 \times 50 \times 80 \mathrm{~mm}^{3}$ [1]. The instrument is shown in Fig. 1. The spectral resolution of the spectrometer is $6 \mathrm{~nm}$. It employs an Indium Gallium Arsenide (InGaAs) infrared (IR) detector with diffractive optics. An instantaneous field of view (IFOV) 2.18 mrad provides a high spatial resolution pollution monitoring capability for this space based instrument. The device includes a programmable Peltier-effect cooler that enhances noise performance.

\subsection{Instrument Description}

Argus 1000 monitors the greenhouse gases contributing to the current and projected changes in the Earth atmosphere from space. The instrument operates in the near IR band between $900 \mathrm{~nm}$ to $1700 \mathrm{~nm}$ with an instantaneous surface resolution approximately $1.25 \mathrm{~km}$. These characteristics enable the identification of precise location of the pollution sources and their local variations. The IFOV measurement is shown in Fig. 2. The narrow $1.25 \mathrm{~km}$ IFOV is particularly beneficial as it yields more spectra that are not partially contaminated by clouds. Argus 1000 includes a detector array of $1 \times 256$ elements that is actively cooled. Each pixel has a radiometric resolution of 12 bits with exposure time ranging from microseconds to seconds for data recording.

The instrument's functional design includes a microcontroller that controls the device components and acquires the spectra. The command interface accepts commands via prime 


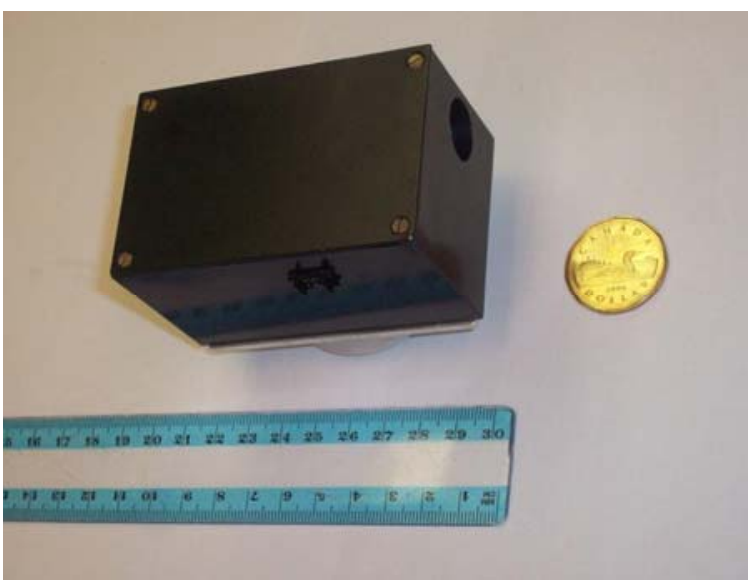

Fig. 1. Argus 1000 Spectrometer.

and redundant serial interfaces and delivers spectra and engineering data via the same interface. The instrument consumes less than $3 \mathrm{~W}$ and includes internal power isolation and regulation. Argus 1000 employs a triplet lens aft optics to discriminate IR radiation onto a spectrally diffracting grating element. Then the signal is focused onto a linear photodiode array with 256 high-quantum efficiency pixels. The instrument's optical configuration is such that 136 pixels are illuminated. This array is a hybrid device with InGaAs and CMOS activepixel readout electronics where the photo-current is buffered, amplified, and stored. The spectral information are differentially sampled in a form of double-correlated sampling, which enables the detection of radiation emitted for $1.56 \mathrm{~km}^{2}$ surface tile to high precision. An order filter, positioned after the aft optics, prevents the visible radiation (below $900 \mathrm{~nm}$ ) from entering the spectrometer chamber.

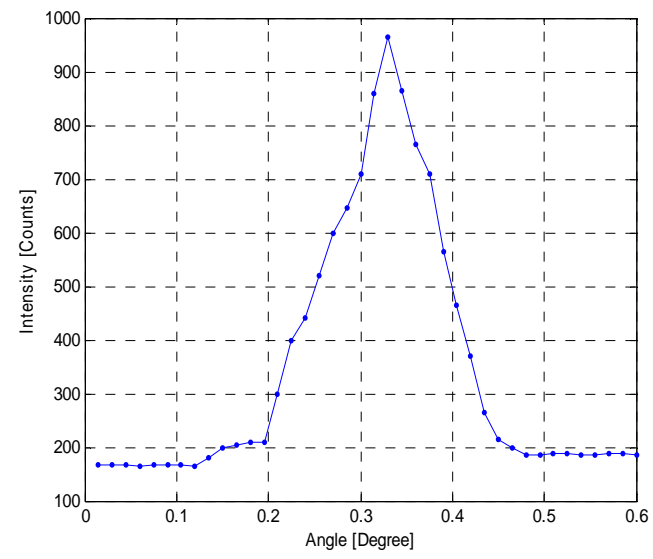

Fig. 2. Angular sensitivity obtained by using milli-Watt collimated laser source at $1064 \mathrm{~nm}$.

The optical layout of Argus 1000 is shown in Fig. 3. Its optical design has a primary parabolic mirror focal length and diameter of $35 \mathrm{~mm}$. A $15 \mathrm{~mm}$ diameter input beam (blue lines) has a focal length of $30 \mathrm{~mm}$. The corresponding F-number is 2.0. The instrument utilizes a plane grating with 300 groves per $\mathrm{mm}$ in the second order to split infrared radiation by wavelength or frequency. 


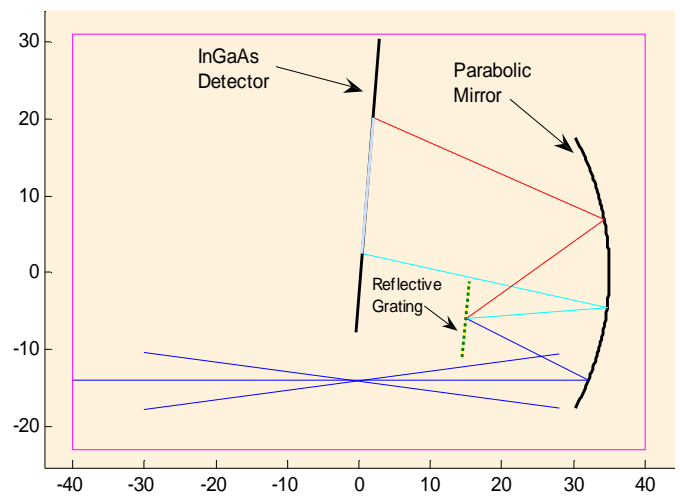

Fig. 3. The optical layout for Argus 1000.

\subsection{Measurement Objectives}

Earth surface reflection spectra contain significant absorption features associated with the molecular absorption of radiation by particular gas species that can be used to infer the composition of the intervening atmosphere [2].

Argus measures the surface-troposphere amounts of the significant greenhouse gases including carbon dioxide $\left(\mathrm{CO}_{2}\right)$, methane $\left(\mathrm{CH}_{4}\right)$, and water vapour $\left(\mathrm{H}_{2} \mathrm{O}\right)$ in order to monitor anthropogenic pollution and to identify significant sources and sinks in the atmosphere. Oxygen $\left(\mathrm{O}_{2}\right)$ absorption features can be utilized to infer column amount. Carbon monoxide (CO) and hydrogen fluoride (HF) species also have absorption features in this spectral region although their signature is significantly weaker. The instrument operates from space to record infrared spectra of the Earth's surface. The spectra are then compared with theoretical IR absorption signatures that model the atmospheric absorption process. In the absence of saturation or scattering effects, the amount of absorption is proportional to the density of the absorber gas along the path. The primary measurement objective of the instrument is to observe any changes in the optical depth, associated with the variation of the identified atmospheric gas species in the spectral interval $900-1700 \mathrm{~nm}\left(11,111-5,882 \mathrm{~cm}^{-1}\right)$. Actual theoretical absorption spectra are computed recursively for each observation scenario by lineby-line GENSPECT radiative transfer code [3].

\section{CALIBRATION PROCEDURE}

\subsection{Spectral and Radiometric Calibration}

Argus 1000 is calibrated in a three-step process, similar to that described in previous works [4-6]. First, a wavelength calibration is conducted using five infrared lasers at 969, 1064, 1150, 1265 and $1523 \mathrm{~nm}$. The spectrometer is mounted on a vernier kinematics mount with five degrees of freedom in order to facilitate angular and spectral calibration.

After the wavelength response is determined, the device is calibrated for the absolute radiometric accuracy using a calibrated standard illumination source, mounted at the distance of $0.5 \mathrm{~m}$ in front of the instrument. The white tile (screen) used in this experimental setup is SRT 99-120. The traceable standard lamp is NIST SRS-99-020-REFL-48. The mean value and standard deviation of the reflectance factor for the white tile are 0.9777 and 0.0141 , respectively.

The spectral irradiance $\left[\mathrm{Wm}^{-2} \mathrm{~nm}^{-1}\right]$ is computed as 


$$
\begin{aligned}
Y & =\frac{1}{\lambda^{5}} \exp \left(A+\frac{B}{\lambda}\right) \\
& \times\left(C+\frac{D}{\lambda}+\frac{E}{\lambda^{2}}+\frac{F}{\lambda^{3}}+\frac{G}{\lambda^{4}}+\frac{H}{\lambda^{5}}\right),
\end{aligned}
$$

where $\lambda$ is the wavelength covering the spectrometer range from 900 to $1700 \mathrm{~nm}$ and $A, B$, $C, D, E, F, G, H$ are corresponding calibration constants, determined with reference to NIST traceable standard for a $10^{3} \mathrm{~W}$ calibrated lamp ORIEL (Model 63350) for the radiance calibration.

The radiant power $[\mathrm{W}]$ entering into the spectrometer can be found as

$$
P=\frac{Y \Omega a \Delta \lambda_{\text {pixel }}}{\pi}
$$

where $a=\pi r^{2}$ and $r=0.0075 \mathrm{~m}$ are the area and radius of the spectrometer lens, respectively, $\Omega$ is the solid angle and $\Delta \lambda_{\text {pixel }}$ is the pixel width in $\mathrm{nm}$.

In order to validate the suitability for launch and reliable operation in space environment, Argus 1000 is subjected to a complete set of space instrument qualification tests including vibration and thermal vacuum cycling.

\subsection{Real-Time Radiance Spectra}

Fig. 4 illustrates spectra, taken over Ontario, Canada, on December 12, 2008 detected by the Argus 1000 from the CanX-2 satellite [7]. This figure shows the absolute radiance measured by the instrument with calibration parameters, derived from our pre-flight calibration of the instrument. Our results appear consistent with other space bound measurements of nadir solar radiance gathered by instrument such as SCIMACHY [8]. From these spectra we can observe the dominant water vapour absorption near $1.4 \mu \mathrm{m}$ and carbon dioxide absorption near $1.42 \mu \mathrm{m}$ and $1.6 \mu \mathrm{m}$. Additionally, we can observe the signature of oxygen around $1.28 \mu \mathrm{m}$. The obtained results clearly indicate that these species are responsible for significant IR absorption in this atmospheric window. Variations in scene intensity are thought likely to be attributable to the presence of clouds in some scenes causing variations in optical depth. Retrieval codes (under development) model for the nadir surface reflected viewing geometry and will also provide estimates of reflected atmospheric radiance, cloud presence and surface albedo.

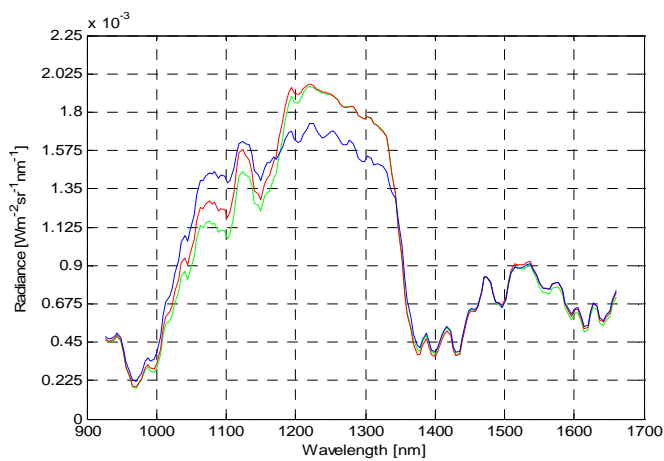

Fig. 4. Spectra taken over Ontario, Canada for Argus 1000. The color lines show three consecutive spectra packets on Dec 12, 2008 from 15:57:32 to 15:57:34 UTC. 


\subsection{Geolocation of Argus Data}

The attitude estimation and control is provided by the by the CanX-2 nanosatellite platform. Attitude estimates are provided to the Argus science team in sixty seconds intervals. The attitude data is interpolated to estimate the attitude at each spectral observation.

Ideally, the observations should be made with the instrument pointing at nadir. However, for timely global coverage it is necessary to be able to acquire spectra at angles up to $\pm 20^{\circ}$ of nadir. Figure 5 shows the nadir angle before and during the 4 minute spectrometer observation period of Fig. 4. As can be seen from Fig. 5, the nadir angle is approximately $7.7^{\circ}$ $\pm 0.5^{\circ}$ for the duration of the observation.

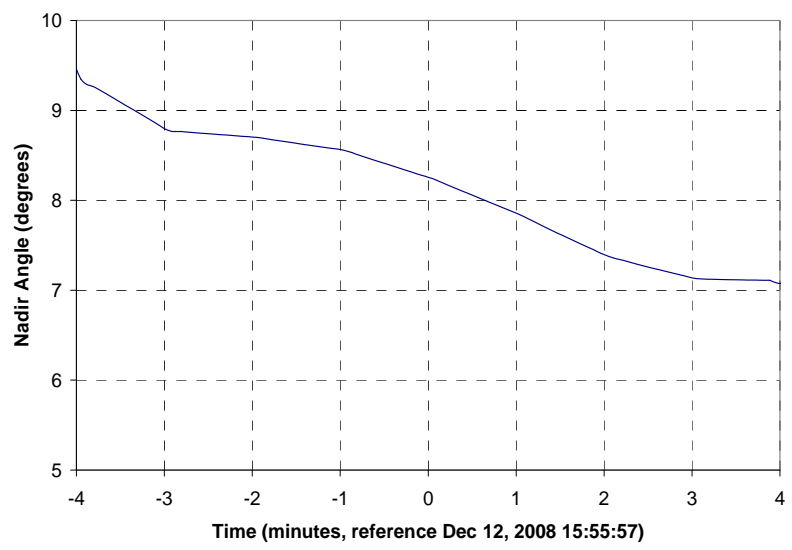

Fig. 5. Argus nadir angle from spacecraft telemetry for same time period as Fig. 4 (time $=0$ is Dec 12, 2008 15:55:57 UTC).

Spacecraft position is estimated using SGP4 propagation of two line elements supplied by NORAD. Using both the position and attitude estimates, Fig. 6 shows the Argus 1000 footprint overlaid on two geographical data sets provided by Google Earth. The points on the ground during the observations of Fig. 4 are shown along with basic geography as well as cloud information that is also required for proper data interpretation. A significant issue for Argus is the availability of local cloud data for each measurement. Pseudo-true colour AVHRR cloud imagery [9] is used to assess the quality of the instrument data. For the particular measurements of Fig. 4, we estimate the geolocation uncertainties along track and cross-track as $22.3 \mathrm{~km}$ and $12.1 \mathrm{~km}$, respectively. In Fig. 6 an ellipse of these uncertainties has been placed around the geolocated measurements of Fig. 4 to give an indication of the uncertainty region.

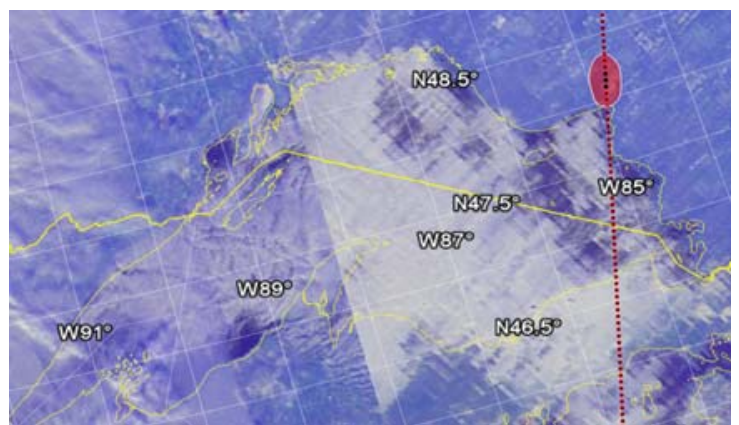

Fig. 6. Geolocated Argus observations using Google Earth with geography and cloud data overlaid. Observations of Fig. 4 are shown as dots with the uncertainty zone in red. 


\section{CONCLUSION}

A small size, light and inexpensive spectrometer was calibrated and tested by the space test facility at York University. The calibration procedure and spectral response function of Argus 1000 is described. With 18 months of orbital heritage on its first flight, the miniature spectrometer meets the requirements for durable operation in orbit. Retrieval of data products from Argus 1000 spectra including differential and absolute column data are in process and will be presented in the forthcoming publications.

\section{Acknowledgments}

This work is supported by the Natural Sciences and Engineering Research Council of Canada, York University and Thoth Technology Inc. The CanX-2 space mission is supported by Defense Research and Development Canada (Ottawa), MacDonald Dettwiler and Associates Space Missions, Dynacon Incorporated, Ontario Centers of Excellence, ETech Division, Canadian Space Agency and Radio Amateur Satellite Corporation (AMSAT). The Authors would like to acknowledge Prof. R. E. Zee and his team, University of Toronto Institute for Aerospace Studies, for facilitating the first flight of Argus 1000 spectrometer and for providing the science and attitude data presented here.

\section{References}

[1] B. M. Quine, R. Jagpal, and A. Ho, "Argus - a Canadian pollution monitor: instrument overview," Proc. 13th Canadian Astronautics Conf. 2006, Canadian Astronautics and Space Institute, Montreal, Quebec (2006).

[2] J. R. Drummond and G. S. Mand, "Measurement of pollution in the troposphere (MOPITT) instrument: overall performance and calibration requirement," J. Atm. Ocean. Tech. 13, 314-320 (1995) [doi:10.1175/15200426(1996)013<0314:TMOPIT>2.0.CO;2].

[3] B. M. Quine and J. R. Drummond, "GENSPECT: a line-by-line code with selectable interpolation error tolerance," J. Quant. Spect. Rad. Trans. 74, 147-165 (2002) [doi:10.1016/S0022-4073(01)00193-5].

[4] T. S. Pagano, H. H. Aumann, D. E. Hagen, and K. Oweroye, "Prelaunch and inflight radiometric calibration of the atmospheric infrared sounder (AIRS)," IEEE Trans. Geosc. Rem. Sen. 41, 265-273 (2003) [doi:10.1109/TGRS.2002.808324].

[5] J. H. Walker, R. D. Saunders, J. K. Jackson, and D. A. McSparron, NBS Measurement Services: Spectral Radiance Calibration, NBS special publication, 102 (1987).

[6] M. Shimada, R. O. Green, and H. Shimoda, "Calibration of advanced visible and near infrared radiometer," IEEE Trans. Geosc. Rem. Sen. 37, 1472-1482 (1999) [doi:10.1109/36.763260].

[7] K. Sarda, S. Eagleson, E. Caillibot, C. Grant, D. Kekez, F. Pranajaya, and R. Zee, "Canadian advanced nanospace experiment 2: Scientific and technological innovation on a three-kilogram satellite," Acta Astronautica 59, 236-245 (2006) [doi:10.1016/j.actaastro.2006.02.054].

[8] M. Gottwald, H. Bovensmann, G. Lichtenberg, S. Noël, A. von Bargen, S. Slijkhuis, A. Piters, R. Hoogeveen, C. von Savigny, M. Buchwitz, A. Kokhanovsky, A. Richter, A. Rozanov, T. Holzer-Popp, K. Bramstedt, J.-C. Lambert, J. Skupin, F. Wittrock, H. Schrijver, and J. P. Burrows., SCIAMACHY, Monitoring the Changing Earth's Atmosphere, pp. 79-84, DLR, Institut für Methodik der Fernerkundung (2006).

[9] AVHRR (Advanced Very High Resolution Radiometer) Google Earth Imagery, downloaded from Space Science and Engineering Center, http://ge.ssec.wisc.edu/avhrr/. 